\title{
Study on Determination Method of Load Measurement Sample Length of the Loader Working Device
}

\author{
Yipin Wan, Jie Jia and Xuding Song \\ The Key Laboratory of Road Construction Technology and Equipment, Chang'an University, Xi'an 710064, China
}

\begin{abstract}
In order to determine the sample size in the load spectrum measure of the loader working device, the methods of the sample length determining were studied. The load test system of the loader working device was constructed and the original load time history data were collected. Considering the load characteristics and the confidence level of the population and the sample data, a new method was proposed and the minimum sample size required for the load test of the loader working device on ZL50 loader was 70 based on the measured load. The results of sample length of different methods were compared and analyzed. The new method presented in this paper was more reliable and credible than other methods. Methods and results to determine the test data is obtained, which provides a reference for the load test and life cycle spectrum compiling.
\end{abstract}

\section{Introduction}

In earthwork construction, working medium and working conditions are complicated and changeable; fatigue failure is the main failure mode of the loader working device [1]. The load time history is the comprehensive performance of the structure in the working environment, which could describe the strength of the structure and the size of the stress [2-3]. Load spectrum is an important foundation for the structural strength analysis and fatigue life reliability analysis of shaft parts. [4], the length of the load sample determines the load spectrum accuracy and load test costs [5]. In general, the larger the load sample capacity, the more accurate the load spectrum data and the fatigue life are obtained. However, too large samples brought about by the high cost of testing, the accuracy of the load spectrum is not obvious. Statistical characteristics of data and error are the theoretical basis for determining the length of the sample. In literature [6], the sample length is studied, and the influence of sample size on the model parameter estimation is defined, and the basic selection principle of the minimum and maximum sample length is given. The [7] analysis method to determine the error meet the required sample length from two aspects of amplitude and frequency, given power spectral density to determine the sample length of the theoretical calculation formula. The [8] in the index model is based on the coefficient of variation of sample length of random data were calculated; the literature [9] considering the statistical accuracy, the accuracy of approximate mean value formula for determining the length of the sample estimate; literature using [10-11] method to fit the trend line of the obtained load test sample length.

Minimum sample size is the key foundation for the testing and compilation of load spectrum. Taking the domestic 50 type loader as the research object, the load signal acquisition system of the loader working device is constructed. In order to test the data as the foundation, proposed a method to determine the actual load of the loading shaft parts based on the length of the sample, considering a variety of statistical characteristics of the actual load data, is given to meet the given error precision of the loading shaft parts minimum sample size, which provides a reference for the loader working device load test method and load spectrum compiling.

\section{Methods for determining the number of load samples}

It is the basic principle that the sample size is determined by the statistical characteristics of the sample data [12], and the minimum length of the sample is obtained within the error tolerance range. For shaft loaders parts load parameters, which are stochastic data obtained by a stable physical phenomenon, could be considered as having characteristics of stationary ergodic [13]. Commonly used sample length determination methods are spectral density method, approximate mean estimation method and trend line fitting method.

The spectral density method is based on the time of the load cycle and the approximate solution of the formula (1).

$$
\varepsilon_{1} \approx 1 / \sqrt{B_{\mathrm{e}} T}
$$


Approximate mean value estimation method, the parameter load time history data are considered as random variables. From statistical knowledge, the overall mean and standard deviation could not be obtained. But we could use a certain length of the sample mean and standard deviation to replace the overall, at this time could be approximated by a mathematical model of the mean estimation method (2).

$$
\varepsilon_{2}=\sigma_{x} /\left(\sqrt{K} u_{x}\right) \approx s_{x} /(\sqrt{K} \bar{x})
$$

In the above formulas, $\varepsilon_{1}$ is the spectral density statistics error; $B_{\mathrm{e}}$ is the frequency band width; $T$ is the sample time length under the statistical error. $\varepsilon_{2}$ is the statistical error of the approximate mean estimator; $K$ is the number of samples under a given statistical precision; $\sigma_{x}$ and $u_{x}$ are the overall standard deviation and the mean, respectively; $s_{x}$ and $\bar{x}$ are the sample standard deviation and the mean value, respectively.

In a given statistical accuracy and sample mean and standard deviation, we could calculate the statistical error of the sample size required to test the test. Trend line fitting method is based on a large number of experimental data, analysis of the parameters of discrete data. To increase the sample length and calculate the mean value of different samples, the mathematical model which is closest to the change trend of the parameters is selected to fit the changing process, so that the variation trend of these parameters with the length of the sample is obtained. When the length of the sample is enough, we could use the sample to replace the overall parameter, and then determine the length of the sample with the statistical precision. According to the trend of the finite sample capacity data, the data fit the exponential model or the hyperbolic model, and the length of the sample is the minimum sample size when the fitting curve is stable.

In general, the sample variance is used to replace the total variance. From the central limit theorem, we could know that the random variable $\bar{x}-u_{x} /\left(s_{x} / \sqrt{n}\right)$ obeys the $t$ distribution. The probability relationship between the significant level and the number of samples from the population is shown in the formula (3).

$$
P\left\{\left|\bar{x}-u_{x}\right| \leq\left(s_{x} / \sqrt{n}\right) t_{\alpha / 2}(n-1)\right\}=1-\alpha
$$

The confidence interval of the sample mean value could be got at this time for a given confidence level of 1- $\alpha$ as shown in the formula (4).

$$
\bar{x}-\left(s_{x} / \sqrt{n}\right) t_{\alpha / 2}(n-1) \leq u_{x} \leq \bar{x}+\left(s_{x} / \sqrt{n}\right) t_{\alpha / 2}(n-1)
$$

$V_{x}=s_{x} / u_{x}$ as the coefficient of variation; $\varepsilon_{3}=\left(u_{x}-\bar{x}\right) / u_{x}$ is the relative error of the overall mean and the sample mean, and the lower the relative error of the overall parameters of the estimated accuracy is higher. The relationship between the coefficient of variation and the relative error is shown in the formula (5).

$$
\varepsilon_{3}=1-V_{x} \cdot \bar{x} / s_{x}
$$

At this time, the relationship between the relative error and the length of the sample could be obtained. The model is shown in the formula (6).

$$
\varepsilon_{3} \geq\left|\left(s_{x} / u_{x} \sqrt{n}\right) t_{\alpha / 2}(n-1)\right|=\left|\left(V_{x} / \sqrt{n}\right) t_{\alpha / 2}(n-1)\right|
$$

Putting the formula (5) into the formula (6) could have the formula (7) as follows.

$$
n \geq\left[\left(\left(1-\varepsilon_{3}\right) / \varepsilon_{3}\right) \cdot \mathrm{s}_{x} / \bar{x} \cdot t_{\alpha / 2}(n-1)\right]^{2}
$$

For a given confidence level, according to the sample mean and standard deviation and the formula (7) could calculate the minimum sample size required for a given statistical error under. However, the sample size is unknown in the practical solution, and the $t_{\alpha / 2}\left(n_{1}-1\right)$ is unknown at this time. Therefore, it is required to determine the size of the $t_{\alpha / 2}\left(n_{1}-1\right)$ according to the initial value of the selected sample number. By the formula (7) a new sample length, calculated the number of samples for recycling, until by $t_{\alpha / 2}\left(n_{1}-1\right)$ and formula (7) the number of samples to obtain consistent loop termination.

\section{Load testing and data processing for loader working device}

The loading shaft parts including load test and axle shaft torque and rotational speed and real time shift information. The stress characteristics of the working device of the loader are analyzed. The axial force of each pin is the basic data of the structural design and fatigue life analysis of the working device. Select the corresponding test parameters to be tested, build the load spectrum test system of work device as shown in Figure 1. 


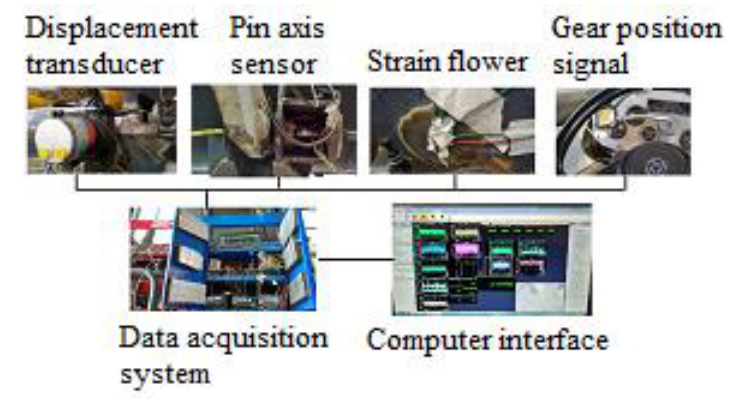

Fig. 1. The load spectrum testing system of the loader working device.

Select the bulk materials containing iron ore particles, with the highest shovel loading efficiency as the principle of shovel loading test. Select the driver with a wealth of driving experience, shovel loading route using commonly used $\mathrm{L}$ type line, material loading test as shown in Figure 2.

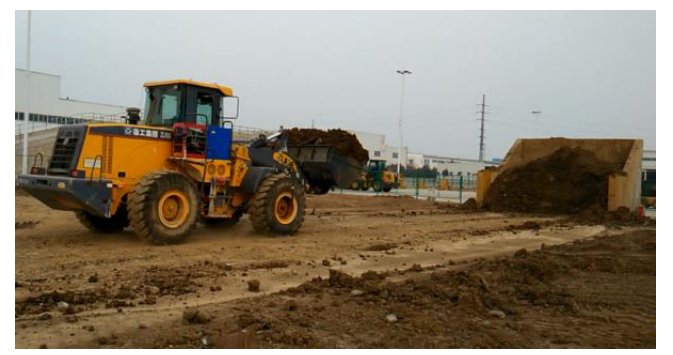

Fig. 2. The load spectrum testing system of the loader working device.

In the measured signal, the interference signal and the peak value of the interference signal caused by the surrounding environment and road surface are included. Preprocessing includes filtering, eliminating trend items, and abnormal peak points. The loading shaft parts load signal for low frequency signal, using $2 \mathrm{~Hz}$ low pass filter. Trend elimination is implemented using Matlab programming. Anomaly points are proposed by using the gradient threshold method to recognize the singular point. After the pretreatment of loader front axle shaft of a torque load signal as shown in Figure 3.

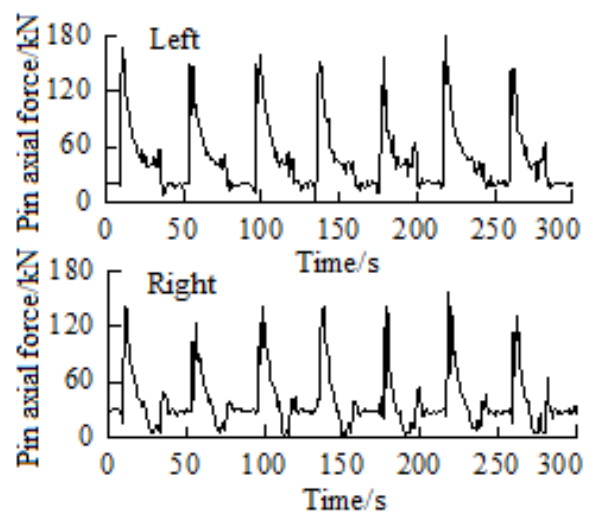

Fig. 3. Load test results of the pin axial force.

We could know from Figure 3 that the measured data of the pin axial force load time history curve shows significant seasonal changes could reflect the load time history of the loader working device assembly process in shovel real stress characteristics. The test data to 5 bucket for a sample, the test data of the 120 bucket, the mean value of the 24 sample data is shown in Table 1.

Table 1. Table of the mean changes in sample data.

\begin{tabular}{|c|c|c|c|}
\hline Sample & Mean/kN & Sample & Mean/ kN \\
\hline
\end{tabular}




\begin{tabular}{|c|c|c|c|}
\hline number & & number & \\
\hline 1 & 51.89 & 13 & 48.69 \\
\hline 2 & 50.48 & 14 & 48.90 \\
\hline 3 & 50.94 & 15 & 48.73 \\
\hline 4 & 50.24 & 16 & 48.70 \\
\hline 5 & 49.11 & 17 & 48.49 \\
\hline 6 & 49.41 & 18 & 48.38 \\
\hline 7 & 48.89 & 19 & 48.28 \\
\hline 8 & 48.64 & 20 & 48.59 \\
\hline 9 & 48.93 & 21 & 48.91 \\
\hline 10 & 48.52 & 22 & 48.92 \\
\hline 11 & 48.25 & 23 & 48.72 \\
\hline 12 & 48.20 & 24 & 48.83 \\
\hline
\end{tabular}

\section{Result analysis}

The average time for a shovel loading test cycle is $45 \mathrm{~s}$, by the formula (1) could get the result of the spectral density method is about $16000 \mathrm{~s}$, that is, 350 buckets. By using the method of approximate mean value estimates, measured 120 bucket sample data calculation of the sample mean is $48.83 \mathrm{kN}$, the sample standard deviation as $3.48 \mathrm{kN}$, the statistical error of 0.05 , by the formula (2) could get the required number of samples is rounded up to 17 . Each sample contains 5 buckets, and the sample length is approximately 105 .

Statistical analysis of the measured data by using the trend line fitting method. Describe the change trends of the mean of Table 1 with the increase of sample number, respectively, with the exponential model, polynomial model and $\mathrm{S}$ curve model to fit the trend of the data, the fitting trend line as shown in Figure 4

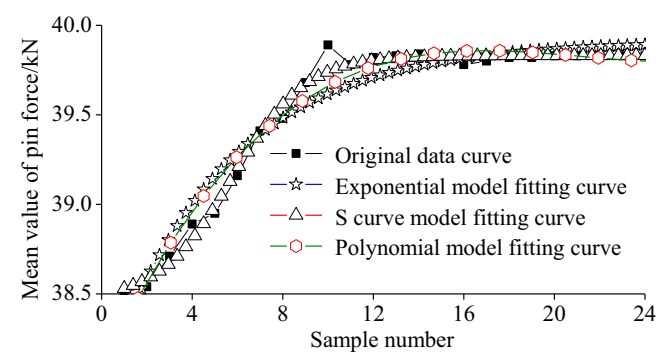

Fig. 4. Schematic diagram of the trend line fitting method.

Figure 4 shows the mean value of the sample, the exponential model of the fitting curve of the goodness of fit is 0.94, the fitting equation as shown in the formula (8).

$$
M=-0.0009 n^{3}+0.0471 n^{2}-0.7620 n+52.36
$$

The goodness of fit curve of polynomial model is 0.95 , and the fitting equation is as shown in formula (9).

$$
M=4.603 \mathrm{e}^{-n / 3.057}+48.57
$$

The goodness of fit of the $\mathrm{S}$ curve model is 0.92 , and the fitting equation is as shown in the formula (10).

$$
M=48.61-3.863 /\left(1+\mathrm{e}^{(n-3.042) / 1.643}\right)
$$

The required sample sizes of the three models are 17, 19 and 15, respectively. The sample length is 85,95 and 75 , respectively. With the curve fitting method, the results are quite different with the different models.

By using the method that sample length calculation based on measured load data proposed in this paper, the iterative calculation is realized by computer programming. For a given confidence level is 0.95 , the statistical error is 0.05 , the initial sample selection is 20 . According to the formula (6) and the iterative procedure described in this paper, the output value of the sample is calculated in the range of 70 75. With the increase of the initial value of the sample length, the length of the output sample is gradually stabilized, and the sample length is 70 .

From the above results, the trend line fitting method results in dependence on the selected model is too strong, the fitting results contain too many artificial factors, the length of the sample results of lack of credibility. The mean value and the standard deviation of the sample load are obtained by using the approximate mean value estimation method, and the result of the sample size is 105 . The proposed estimation method based on the central limit theorem, consider not only the mean and standard deviation of measured load characteristics of sample loading, but also consider the overall mean probability falls on the sample mean interval is the confidence level, makes the results more reasonable. The 70 
bucket sample size obtained by this method can be considered as the minimum sample size required to be tested, and the model parameters are estimated to be stable. The results of the new analysis method proposed by common methods and the minimum length of the sample shows that the load testing required is 70 .

\section{Conclusion}

In this study, the method of determining the sample in the load test of the loader working device is studied, and the following conclusions are obtained.

According to the loading characteristics of the loader working device, the load test system was constructed. The load sample data of loading test reflect the real operation characteristics of loader working device, load sample length determination method to determine the basis. The method of determining the length of the load sample is analyzed. The sample length estimation method based on the measured load data is proposed. The method not only considers the characteristics of sample loading and standard deviation, but also considers the confidence level of the population mean and the sample mean, and the result is more reliable and credible than the other methods. The load test samples required minimum length is 70 , and the research results provide an important reference spectrum and fatigue life prediction of the loader working device. The next step is to increase the amount of data, and further study the effect of sample size on the results of the load spectrum and the results of fatigue reliability analysis.

\section{Acknowledgements}

In this paper, the research was sponsored by the National Key Technology Research and Development Program of the Ministry of Science and Technology of China (Project No. 2015BAF07B02) and the Fundamental Research Funds for the Central Universities (No. 310825163407).

\section{References}

1. J. Samuelsson, Jonsson B, Barsoum B. Service fatigue design of complex welded construction equipment. Material-wiss. Werkstofftech 10, 734-739 (2008).

2. H. Guo, W.H. Chen, X.Y. Fan. Research of enhancement coefficient of automobile reliability enhancement test on proving ground. Chinese Journal of Mechanical Engineering 10, 102 (2004).

3. S. Murer, D. Leguillon. Static and fatigue failure of quasi-brittle materials at a V-notch using a dugdale model. European Journal of Mechanics A/Solids 8, 109-118(2010).

4. J.X. Wang, J. Hu, X.T. Hou. Loader axle shaft finite element analysis based on load spectrum. Modern Manufacturing Engineering 5, 1-3(2010).

5. C.H. Yan, Z.T. Gao. Design criterion of the flight takeoff-landing number of minimum no-loading measurement sampling. Acta Aeronautica Et Astronautica Sinica 20, 514-517(1999).

6. P.X. Gao. Selection of sample length for parameter modeling. Journal of Vibration, Measurement and Diagnosis 4 , 5-10 (1991).

7. L.D. Shi. Finite life design and testing of machinery Seventh speak: the development process of the load spectrum. Construction Machinery 10, 35-45 (1986).

8. L.D. Shi, J.G. Zhang, S.Q. Huang. Discussion on the problem of determining the length of the sample in the process of random data processing. Construction Machinery 4, 16-21 (1993) .

9. Y.L. Zhang, W.N. Chu, C.X. Xu. Determination of load sample length for loader driveline. Construction Machinery and Equipment 6, 16-20 (1994).

10. C.H. He, H. Zhou, J. Xiang. Study on the method of load spectrum sample size determination in vehicle road testing. Automobile Technology 11, 14-17 (2003).

11. Y.N. Li, S.F. Lin, S.H. Liu. Application of regression analysis to sample size's error analysis. Journal of Jimei University 1, 72-75 (2010).

12. D.L. Cheng, J.B. Adam, D.S. James. A Bayesian approach to sample size determination in mechanical engineering. Computational Statistics and Data Analysis 54, 298-307 (2010).

13. Y.P. Wan, J. Jia, J. Liang. Structural strength analysis and experimental research for working device of loader. Journal of Mechanical Strength 4, 772-776 (2016). 